\title{
The role of the structural connectome in literacy and numeracy development in children
}

\author{
Joe Bathelt $^{1}$, Susan E Gathercole ${ }^{1}$, Sally Butterfield ${ }^{1}$, Duncan E Astle ${ }^{1,}$ \\ and the CALM Team
}

${ }^{1}$ MRC Cognition and Brain Sciences Unit

15 Chaucer Road

Cambridge CB2 7EF

Corresponding author:

Dr. Joe Bathelt (joe.bathelt@mrc-cbu.cam.ac.uk) 


\begin{abstract}
Literacy and numeracy are fundamental skills acquired in childhood, a time that coincides with considerable shifts in large-scale brain organisation. However, most studies emphasize focal brain contributions to literacy and numeracy development by employing case-control designs and voxel-by-voxel statistical comparisons. This approach has been valuable, but does not capture the potential importance of broader differences in brain organisation. The current study aims to address this by including children with varying levels of reading and maths ability, and by using a whole-brain structural connectome approach based on diffusion-weighted MRI data. Our results indicate an association between literacy and numeracy development and a distributed network of white matter connections that extends well beyond regions implicated in voxel-wise studies. Graph theory measures of network organisation were predictive of reading and maths scores. Simulated disruption of highly-connected hubs indicated that these regions are particularly important for optimal network organisation. These findings show that changes in large-scale brain organisation contribute to improvements in literacy and numeracy as children grow up.
\end{abstract}

Keywords: Mathematics, Reading, Brain Development, White Matter, Connectome 


\section{Introduction}

Studies of the structural basis of inter-individual variation in reading and maths abilities have so far laregely emphasised particular anatomical substrates (Peterson and Pennington 2015, K Kucian and M von Aster 2014). These are largely derived a priori from adult neuropsychological models or from the use of statistical procedures optimised to uncover maximal focal differences in canonical case-control designs (Carter et al. 2009, Rollins et al. 2009, Odegard et al. 2009, Matejko et al. 2013, Kucian et al. 2013, Beek et al. 2014). While these approaches have been invaluable for discovering the brain basis of acquired adult disorders, their applicability to disorders of developmental origin has been questioned for some time (Karmiloff-Smith 1998). In contrast to classical adult neuropsychology, in which specific functions are mediated by specific brain modules, recent theoretical approaches suggest that specialization arises from the interaction between brain regions over the course of development (Johnson 2003, Pascual-Leone et al. 2005). As a result of these interactions, developmental cognitive problems are likely to be associated with changes in brain organisation, as problems cascade through the system or are partially compensated for elsewhere.

The approach typically taken to understanding the neural basis of developmental cognition is unlikely to capture these developmental cascades for several reasons. Firstly, the use of voxel-wise statistical comparisons emphasizes the small number of voxels of overlap across children, and ignores any broader differences in brain organisation. Secondly the use of case-control designs with the strict selection of cases and controls can give a misleading appearance of the relative purity of any cognitive deficit (e.g. (Ranpura et al. 2013)). In reality, comorbidity is more the rule than the exception in developmental disorders (Larson et al. 2011, Pennington and Bishop 2009, Landerl and Moll 2010, Gillberg 2010), but the real-world presentation of these difficulties is often not reflected by the cohorts of children studied. Together these design choices can give the false impression that developmental cognitive disorders arise from focal 'lesion-like' brain differences.

In these traditional neuroimaging studies (Peterson and Pennington 2015, Kucian and von Aster 2014), deficits in these aspects of learning has been linked differences in tracts of the language system, including the inferior and superior longitudinal fasciculus/arcuate fasciculus, posterior corpus callosum, and extreme capsule (Carter et al. 2009, Rollins et al. 2009, Odegard et al. 2009). In the case of mathematical abilities, white matter connections of the parietal and frontal lobe have been implicated (Matejko et al. 2013, Kucian et al. 2013, Beek et al. 2014). 
The approach to understanding the relationship between white matter organisation and the development of literacy and numeracy adopted in the present study differed in two key respects from previous research. First, a large sample of children $(n=88)$ was recruited with mathematics and reading abilities spanning the full range from impaired to age-appropriate performance levels. Individuals were not excluded on the basis of comorbid neurodevelopmental disorders, yielding a sample that reflects the typical presentation of these cognitive disorders in the population (Landerl and Moll 2010, Kovas et al. 2007).

Second, we applied contemporary network science methods to provide a comprehensive account of the neuroimaging data. In a network analysis, brain regions are described as nodes and their connections as edges. Nodes typically correspond to regions of interest (Dell'Acqua and Catani 2012, Fornito, Zalesky, and Breakspear 2015). Edges can represent the strength of white matter connectivity based on diffusion-weighted imaging (Qi et al. 2015). Graph theory provides a mathematical framework for the analysis of the resulting network (Bullmore and Sporns 2009, Rubinov and Sporns 2010), which describes organisational principles related to ease of information exchange and wiring cost. Typical childhood is characterised by increases in global efficiency between preschool years and adulthood (Hagmann et al. 2010, Fan et al. 2011, Yap et al. 2011, Dennis et al. 2013, Huang et al. 2013, Khundrakpam et al. 2012, Wierenga et al. 2015, Hagmann et al. 2010, Dennis et al. 2013, Huang et al. 2013). In contrast, structural brain networks in atypically developing groups, including autism, premature birth, hypoxic-ischaemic injury, and attention-deficit hyperactivity disorder (ADHD), were found to deviate from this organisation (Pandit et al. 2013, Pannek et al. 2013, Shi et al. 2013, Konrad and Eickhoff 2010). A set of highly-connected nodes known as the "rich club" is thought to be particularly important for efficient brain network organisation (van den Heuvel et al. 2012, van den Heuvel and Sporns 2011, Crossley et al. 2014). Neurodevelopmental and neurodegenerative diseases were found to show disproportionate anatomical abnormalities in these rich club regions (Crossley et al. 2014) indicating that they may be especially important for understanding atypical brain development (Di Martino et al. 2014).

To investigate how the organisation of the structural brain networks relates to literacy and numeracy development, we constructed a network that represents white matter connections throughout the brain based on diffusion-weighted MRI data. Network-based statistics were applied to assess how clusters of white matter connections related to reading and maths ability scores. Next, the relationship between global measures of network organisation and ability scores, and how this relationship may be governed by highly connected hub nodes was investigated. Finally, the extent to which age-related 
improvement in literacy and numeracy may be explained by the concurrent development of the structural brain network was estimated. 


\section{Participants and Methods}

\section{Participants}

All children participated in a large-scale study at the MRC Cognition and Brain Sciences, the Centre for Attention, Learning, and Memory (CALM) research clinic. At the clinic, children were recruited on the basis of ongoing problems in attention, learning, and/or memory in school and were identified by professionals working in schools or specialist children's community services. Families were invited for an assessment that lasted approximately 3 hours. The assessment included the academic attainment measures reported here. Exclusion criteria for referrals were significant or severe known problems in vision or hearing that were uncorrected, conditions of known genetic origin, and having a native language other than English. This study was approved by the local NHS research ethics committee (Reference: 13/EE/0157). Written parental consent was obtained and children provided verbal assent.

Complete MRI scans were available from 139 children. 27 children were excluded because of missing data on the WIAT Numerical Operations or WIAT Word Reading subtest, 24 children were removed due to excessive movement during the diffusion sequence (maximum frame-by-frame displacement $>3 \mathrm{~mm}$ ). The final sample consisted of 88 participants (33 female; Age [years]: mean=9.51, $\mathrm{SD}=2.306$, Range:6-18).

\section{Assessment of learning}

Children completed the Numerical Operations and Word Reading subset of the Wechsler Individual Achievement Test-Second UK Edition (WIAT-IIUK, Wechsler 2005) as part of a larger battery of tests in an individual assessment setting with a trained researcher. For the Numerical Operations subtest, children had to solve arithmetic problems in a booklet that ranged from basic counting to more complex operations including multi-digit multiplication and calculations with fractions. For the Reading subtest, the child had to read single words from a card that got progressively more difficult and correct pronunciation was scored. For the tests, correct responses were scored until 6 or 7 consecutive scores of zero, respectively, were reached following the reference manual of the test (Wechsler 2005).

At the group level, ability scores in maths and reading were in the low range (Agenormed standard scores: maths: mean $=86.92$, $\mathrm{SE}=1.883$, reading: mean $=87.25$, $\mathrm{SE}=1.827$ ) with $45 \%$ of participants scoring 1 standard deviation or more below the norm mean on maths and $49 \%$ on reading. There was no significant difference between 
maths and reading standard scores (Paired-sample t-test: $\mathrm{t}(87)=-0.19, \mathrm{p}=0.85$ ). Ten children had a specific maths deficit (Age-standardised scores: maths $<85$, reading $>$ 90), 8 children had a specific reading deficit (reading $<85$, maths $>90$ ), 27 children had maths and reading deficits (maths $<85$, reading $<85$ ), and 22 children had no maths or reading deficit (maths $>90$, reading $>90$ ). These scores indicate that the current sample showed a spread of ability in reading and maths ranging from learning deficits in either or both domains to age-appropriate ability. The scores also illustrate the high comorbidity of poor literacy and numeracy skills, which was further reflected in a high correlation between maths and reading scores $(\mathrm{n}=88$, Pearson- $\mathrm{R}=0.74, \mathrm{p}<0.001)$
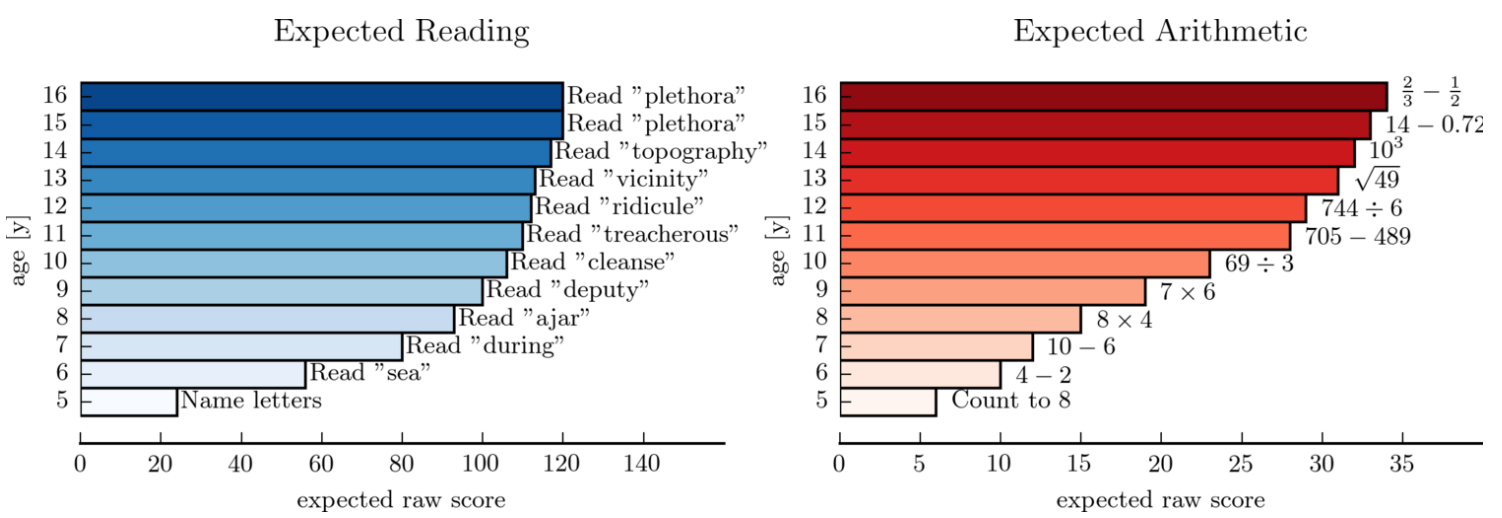

Figure 1: Illustration of sample test items required to reach age-appropriate scores at different ages on the Wechsler Individual Achievement Test 2nd edition UK (WIAT-II UK) Word Reading task (left) and the Numerical Operations task (right). Skills assessed on the attainment measures ranged from basic fact retrieval to complex skills including being able to read non-phonetic words and solving of multi-step calculations.

\section{MRI data acquisition}

Magnetic resonance imaging data was acquired at the MRC Cognition and Brain Sciences Unit, Cambridge U.K. All scans were obtained on the Siemens 3 T Tim Trio system (Siemens Healthcare, Erlangen, Germany), using a 32-channel quadrature head coil. The imaging protocol consisted of two sequences: T1-weighted MRI and a diffusionweighted sequence.

T1-weighted volume scans were acquired using a whole brain coverage 3D Magnetisation Prepared Rapid Acquisition Gradient Echo (MP-RAGE) sequence acquired using 1mm isometric image resolution. Echo time was $2.98 \mathrm{~ms}$, and repetition time was $2250 \mathrm{~ms}$.

Diffusion scans were acquired using echo-planar diffusion-weighted images with an isotropic set of 60 non-collinear directions, using a weighting factor of $b=1000 \mathrm{~s}^{*} \mathrm{~mm}^{-2}$, 
interleaved with 4 T2-weighted $(\mathrm{b}=0)$ volumes. Whole brain coverage was obtained with 60 contiguous axial slices and isometric image resolution of $2 \mathrm{~mm}$. Echo time was 90 ms and repetition time was $8400 \mathrm{~ms}$.

\section{Connectome construction}

The white-matter connectome reconstruction followed a standard procedure of estimating the most probable white matter connections for each individual, and then obtaining measures of fractional anisotropy (FA) between cortical regions after transformation to common space (Horn 2016, see Figure 2). The details of the procedure are described in the following paragraphs. The code for the connectome construction and all analyses detailed in this manuscript are available online (https://github.com/joebathelt/Learning_Connectome).

In the current study, MRI scans were converted from the native DICOM to compressed NIfTI-1 format using the dcm2nii tool developed at the McCauseland Centre for Neuroimaging http://www.mccauslandcenter.sc.edu/_mricro/mricron/dcm2nii.html. Subsequently, the images were submitted to an implementation of a non-local means denoising algorithm (Coupe et al. 2008) in the Diffusion Imaging in Python (DiPy) v0.8.0 package (Garyfallidis et al. 2014) to boost signal to noise ratio. Next, a brain mask of the b0 image was created using the brain extraction tool (BET) of the FMRIB Software Library (FSL) v5.0.8. Motion and eddy current correction were applied to the masked images using FSL routines. The corrected images were re-sliced to $1 \mathrm{~mm}$ resolution with trilinear interpolation using in-house software based on NiBabel v2.0.0 functions (URL: http://nipy.org/nibabel/). A spherical constrained deconvolution (CSD) model was fitted to the 60 gradient direction diffusion-weighted images using a maximum harmonic order of 8. Correct anatomical orientation of CSD glyphs was visually inspected for white matter tracts of known orientation (corpus callosum, cortico-spinal tract).

A whole-brain tractography was generated by seeding streamlines from each voxel within the brain mask using the probabilistic tractography algorithm of MRTRix (Tournier, Calamante, and Connelly 2012). The desired number of streamlines was set to 150,000. Other settings followed the recommendations for MRTrix: The fibre tracking algorithm was set to a minimum and maximum track length of $10 \mathrm{~mm}$ and $200 \mathrm{~mm}$ respectively. The minimum radius of curvature was set to $1 \mathrm{~mm}$ and the track size to $0.2 \mathrm{~mm}$. The track termination threshold was set to an FA value of 0.1 .

Subsequently, a 12 degree-of-freedom affine transform between each participant's skullstripped FA image and the MNI152 template with the correlation ratio as the cost function was calculated using FSL FAST. This transform was applied to the streamlines in each participant's anatomical space to move them into MNI common space (Horn 
2016). For structural connectome analysis, regions of interests (ROIs) were based on the Automatic Anatomical Labelling (AAL) atlas template 10.1006/nimg.2001.0978 with 116 ROIs. The ROIs filled the space between the cortical grey and white matter so that streamlines would terminate at the edges of the ROI. For each pairwise combination of ROIs, the number of streamlines intersecting both ROIs was estimated and transformed to a density map. For FA-weighted networks, this density map was binarized and multiplied with the FA map to obtain the FA value corresponding to the connection between the ROIs. This procedure was implemented in-house based on DiPy v0.8.0 functions (Garyfallidis et al. 2014). To remove spurious connections in the FA-weighted and streamline density-weighted networks, consensus thresholding was applied so that only connections that were present in more than $60 \%$ of the sample were retained (de Reus and van den Heuvel 2013)

Visualizations of the structural connectome were generated using the BrainNet Viewer toolbox (Xia, Wang, and He 2013). 


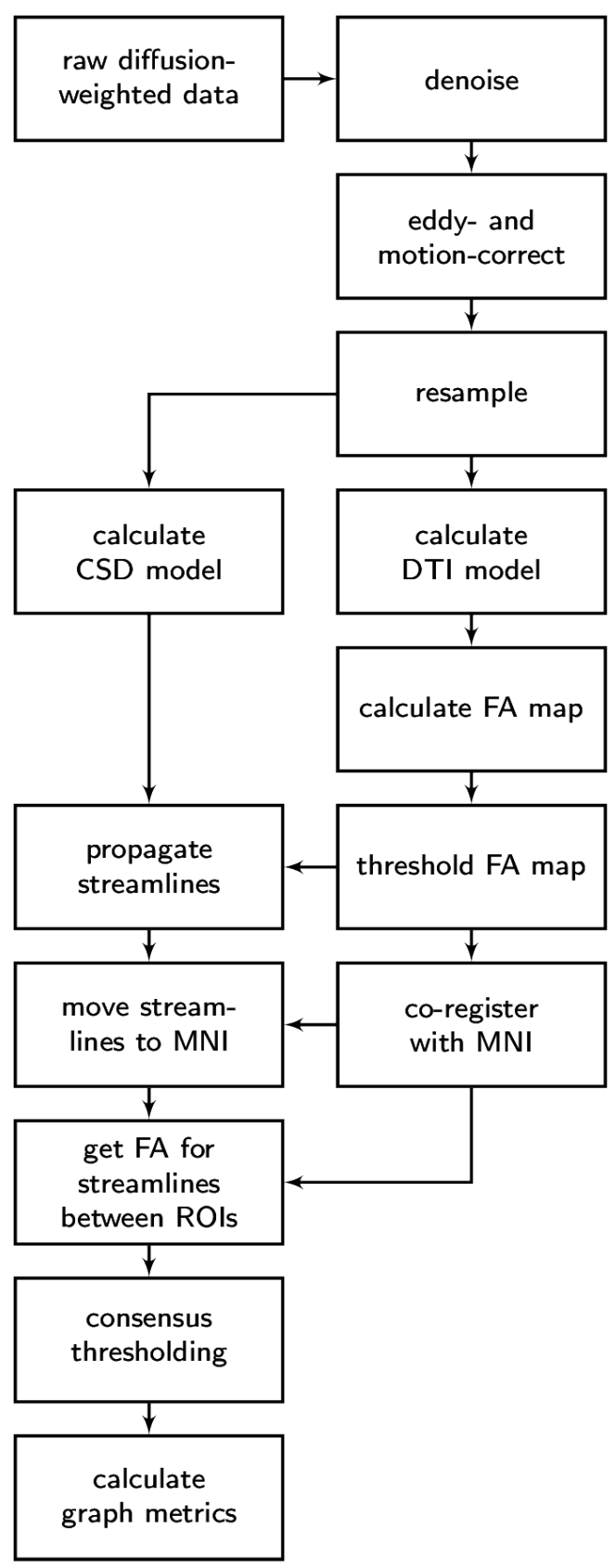

Figure 2: Overview of processing pipeline to construct an FA-based structural connectome description from diffusion-weighted MRI data.

\section{Network-based statistics}

Structural connections that were significantly associated with Reading and Maths performance were identified in a general linear model (GLM). Correction for multiple comparisons was performed using Network-Based Statistics (Zalesky, Fornito, and Bullmore 2010)(NBS, https://sites.google.com/site/bctnet/comparison/nbs). NBS is a method to control the inflation of Type-I errors with multiple comparisons and is akin 
to cluster statistics in canonical statistical parametric mapping. NBS provides greater statistical power than other correction methods like Bonferroni or False Discovery Rate correction. A t-statistic statistic threshold of 3 was used for individual connections (Zalesky, Fornito, and Bullmore 2010), which were subsequently included in permutation testing with 10,000 permutations. An NBS-corrected significance level of $p<0.05$ was used to report results of this analysis.

\section{Graph theory analysis}

The organisation of the structural network $(G)$ was characterised through the average clustering coefficient $\left(C_{G}\right)$ and the characteristic path length $\left(L_{G}\right.$, Rubinov and Sporns 2010). For nodes $i, j$, and $h$ with weight $w$ as a subset of all nodes $N$ in a network $G$ :

The characteristic path length was defined as: $L_{G}=\frac{1}{n} \sum^{i \in N} \frac{\sum^{j \in N, j \neq i} d_{i j}^{w}}{n-1}$ with $d_{i, j}$ as the shortest path between nodes $i$ and $j$ For graph that were not fully connected, $L_{G}$ was calculated for the largest component (Fornito, Zalesky, and Bullmore 2016).

Clustering coefficient was defined as: $C_{G}=\frac{1}{n} \sum^{i \in N} \frac{2 t_{i}^{w}}{k_{i}\left(k_{i}-1\right)}$ with $t_{i}$ as the number of triangles: $t_{i}=\frac{1}{2} \sum^{j, h \in N}\left(w_{i j} w_{i h} w_{j h}\right)^{1 / 3}$

The Brain Connectivity Toolbox for Python implementation was used to calculate these measure (https://github.com/aestrivex/bctpy).

Some external factors may influence graph measure that do not reflect the network property of interest. In the current analysis maximum displacement during the DWI sequence and intracranial volume (ICV) were considered. $C_{G}$ and $L_{G}$ may also be influenced by graph density $\left(D_{G}\right)$. The total number of connections was matched between participants using consensus thresholding, but residual effects may arise from connection weight differences between participants. Multiple linear regression including ICV, movement, and $D_{G}$ indicated a significant effect of $D_{G}$ on $C_{G}$ and $L_{G}\left(C_{G}\right.$ : $\mathrm{F}(3,84)=5.714, \quad p=0.001, \quad \mathrm{R}^{2}=0.17$, Adjusted $\quad \mathrm{R}^{2}=0.14 ; \quad \mathrm{ICV}: \quad \beta=-0.103, \quad p=0.342 ;$ Movement: $\beta=-0.144, p=0.154 ; D_{G}: \beta=0.351, p=0.001 ; L_{G}: \mathrm{F}(3,88)=4.673, p=0.004$, $\mathrm{R}^{2}=0.14$, Adjusted $\mathrm{R}^{2}=0.11$; ICV $: \beta=0.139, p=0.203$, Movement: $\beta=0.155, p=0.130, D_{G}$ : $\beta=-0.2861, p=0.010)$

\section{Simulated attack on connectome nodes}

In order to assess the role of highly connected nodes for the relationship between global graph measures and ability scores, we carried out a simulated attack on highly connected nodes (rich club), peripheral nodes, and randomly chosen nodes. To this end, between one and 20 nodes from each category were selected at random and their 
existing edge weights were knocked-down to the lowest observed value in the groupaverage network, i.e. 0.01. Then, mean $L_{G}, C_{G}$, and $D_{G}$ were calculated in the targeted network. This process was repeated 100 times at each step and the results were averaged to remove effects associated with any particular node. Nodes were not removed completely to keep the number of nodes in the network constant. Different knock-down values $(0.001,0.0001)$ produced similar results to the reported findings. Effects of attack on Maths and Reading scores were based on the predictions for $L_{G}$ and $C_{G}$ in the regression model derived from the empirical data.

\section{Voxel-wise comparison using tact-based spatial statistics}

In order to contrast the structural connectome approach with more commonly used voxelwise statistical analysis, FA maps were processed using tract-based spatial statistics (TBSS) as implemented in FSL v5.0.9. See Smith et al. 2006 for a detailed description of TBSS (Smith et al. 2006). In short, FA maps were moved to common space via an affine and non-linear transformation using FSL tools. A common template, constructed from a large developmental sample by using advanced normalization tools (ANTs) v1.9 (Avants and Gee 2004) was used as the registration target in the current sample (see (Bathelt et al. 2016)). Next, the mean FA image was created and thinned to create a mean FA skeleton which represents the centres of all tracts common to the group. Each subject's aligned FA data was then projected onto this skeleton. For group comparisons, a median split on the age-standardised maths and reading scores was applied. The groups were compared in an independent sample t-test model using a permutation-based algorithm with cluster-free threshold enhancement as implemented in FSL Randomise (Winkler et al. 2014). 


\section{Results}

Mathematics and reading scores are associated with a distributed cluster of connections

In a first step, we investigated how clusters of white matter connections relate to maths and reading ability scores. This was carried out using a general linear model in networkbased statistics with cluster-level correction for multiple comparisons (Zalesky, Fornito, and Bullmore 2010). The results indicated the involvement of a single cluster containing extensive connections between temporal, parietal, and frontal regions within and between hemispheres for both learning scores (see Figure [3). Clusters relating significantly to maths and reading scores comprised a considerable portion of the network edges (7.07\% of all possible connections for maths, $6.23 \%$ for reading).
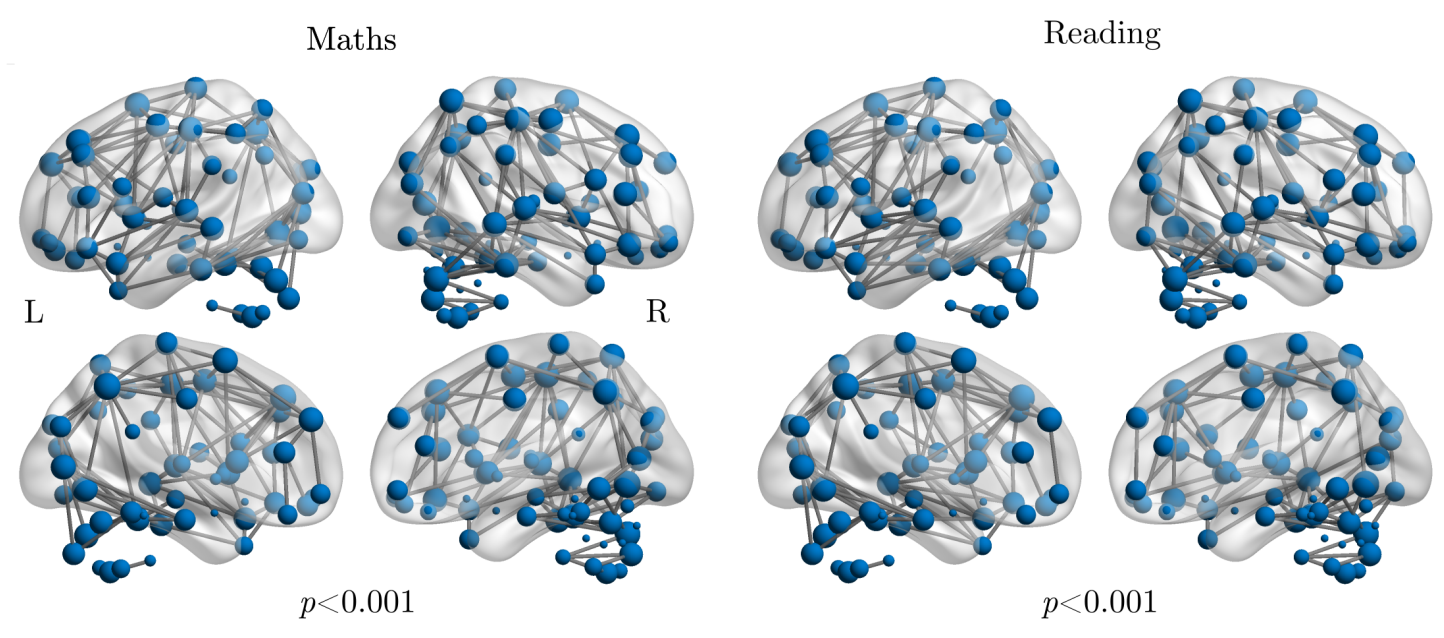

Figure 3:] Analysis of the relationship between maths and reading scores with structural brain connections using network-based statistics (Zalesky, Fornito, and Bullmore 2010). The node degree of each node corresponds to the node size. Only edges that were indicated as being part of a cluster significantly associated with maths (left) or reading scores (right) are shown.

\section{The structural connectome shows small-world organisation}

Next, we aimed to characterise organisational features of the structural brain network as whole to then relate them to maths and reading. To describe organisational features, the observed networks were compared to matched random networks. The random comparison networks are a scrambled version of the original networks to guarantee equal weight and degree distribution. As expected, node strength was identical in the original and scrambled networks (observed: mean $=1.96, \mathrm{SE}=0.015$; random:mean $=1.96$, $\mathrm{SE}=0.015)$. Other graph measures were compared to identify how the observed networks 
differed from random networks. Comparison of graph density indicated higher density in the observed relative to the random networks (Observed density: mean $=0.08, \mathrm{SE}=0.0$, Random density: $\operatorname{mean}=11.5, \quad \mathrm{SE}=0.07 ; \mathrm{t}(85)=44.54, \quad p<0.001)$. Further, the characteristic path length of the observed networks was longer $\left(L_{\text {observerd }}\right.$ : mean $=12.44$, $\left.\mathrm{SE}=0.076, L_{\text {Random }}: \operatorname{mean}=11.5, \mathrm{SE}=0.07 ; \mathrm{t}(85)=44.54, p<0.001\right)$, while average clustering coefficient was higher $\left(C_{\text {Observed }}\right.$ : mean=12.44, $\mathrm{SE}=0.076, C_{\text {Random }}$ : mean $=11.5, \mathrm{SE}=0.07 ; \mathrm{t}(85)=161.67, p<0.001)$. These features indicate small-world organisation of the observed network with longer path length than a random network, but higher clustering.
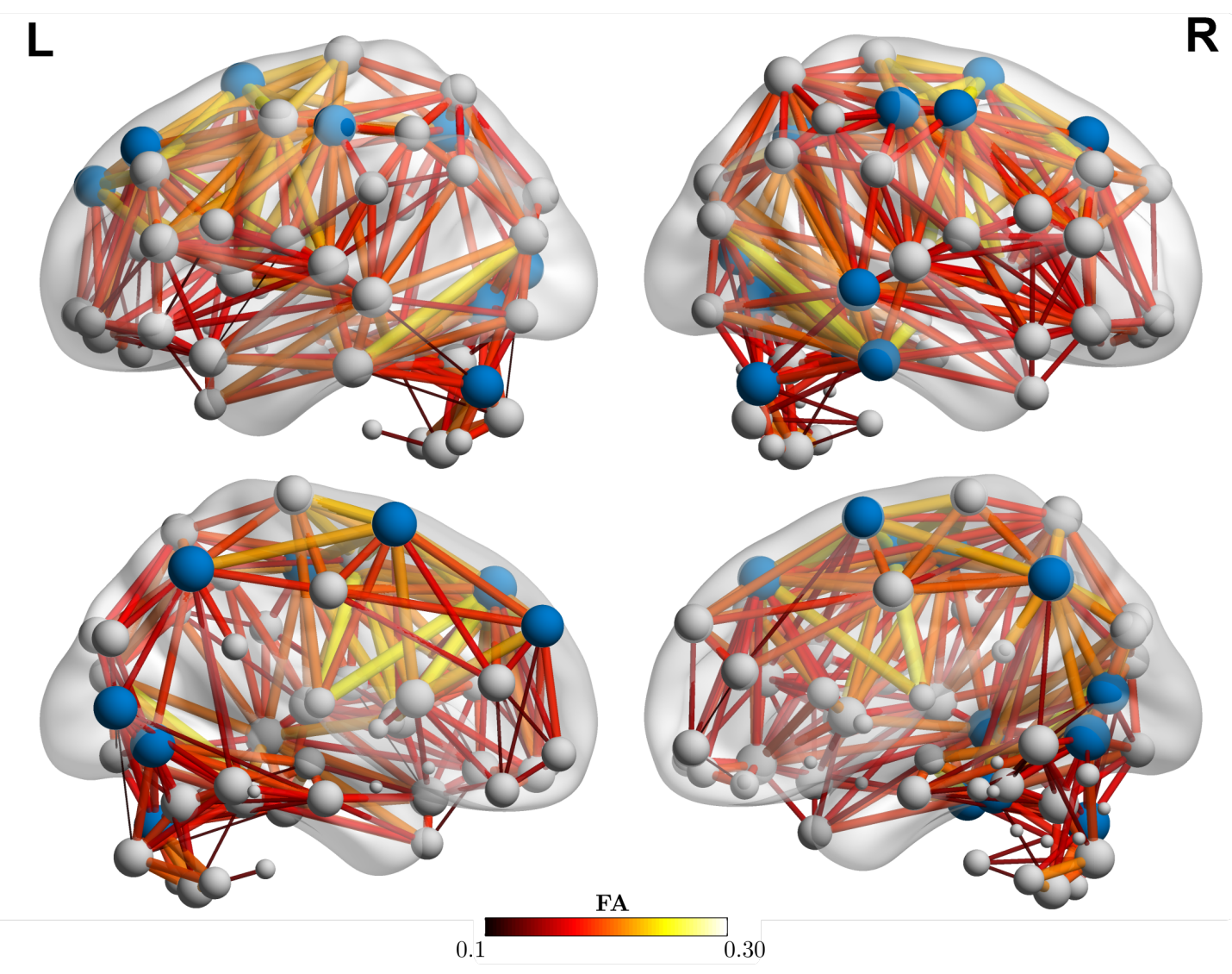

Figure 4: Visualisation of the group-average structural connectome. The left panel shows connections of the left hemisphere in lateral (top) and medial (bottom) view. Similarly, the right panel shows lateral (top) and medial (bottom) connections of the right hemisphere. The middle panel shows a dorsal view of connections. The size and colour of edges correspond to the connection weight (FA). The size of nodes represents the node degree. Nodes shown in blue are nodes of the rich club, i.e. nodes with a degree one standard deviation or more above the mean of all nodes. The group average network was thresholded at 0.1 for visualisation purposes only. 


\section{Small-world organisation relates to reading and maths scores}

Mean clustering coefficient and characteristic path length were used to assess the relationship between academic attainment and structural brain organisation. Linear regression models with reading or maths scores as the outcome and mean clustering coefficient $\left(C_{G}\right)$ or characteristic path length $\left(L_{G}\right)$ as predictors were fitted. Intracranial volume (ICV), maximal displacement during the DWI sequence, graph density $\left(D_{G}\right)$, and an intercept term were also included as regressors of no interest: $Y_{\text {Maths } / \text { Reading }}=$ $\beta_{L_{G} / C_{G}} X_{L_{G} / C_{G}}+\beta_{D_{G}} X_{D_{G}}+\beta_{I C V} X_{I C V}+\beta_{\text {Displacement }} X_{\text {Displacement }}+\beta_{D} X_{D}+\beta_{\text {Intercept }}+\epsilon$

This analysis indicated a significant positive effect of $C_{G}$ on reading and maths scores (Reading: $\beta=0.45 p<=0.001$; Maths: $\beta=0.49, p<0.001$, see Figure 5 ). There was also a significant negative effect of $L_{G}$ (Reading: $\beta=-0.52, p<0.001$, Maths: $\beta=-0.52, p<0.001$ ). In other words, white matter networks of children with better reading or maths scores were more efficient and displayed higher local connectivity.
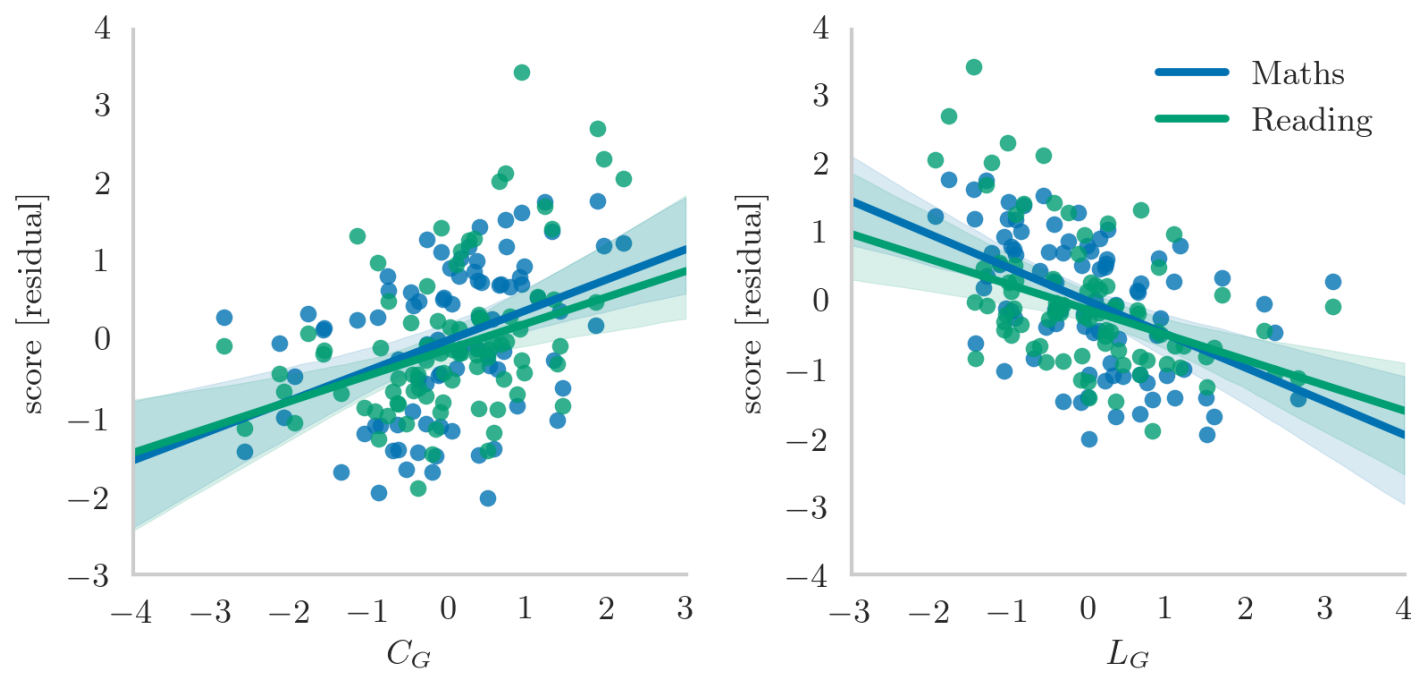

Figure 5: Linear relationship between mean clustering coefficient $C_{G}$ (left), characteristic path length $L_{G}$ (right) and Reading and Maths scores. The Maths and Reading scores shown represent residuals after removing the linear effects of ICV, displacement, and graph density. Graph metrics are expressed as $z$-scores relative to the mean and standard deviation of the whole sample. The shaded area indicates the 95\% confidence interval. Linear regression models indicated a significant positive effect of higher clustering coefficient on Maths and Reading, and a negative effect of higher characteristic path length on both scores. 


\section{The structural connectome contains a rich club of highly connected nodes}

The small-world architecture of human brain networks is accompanied by a set of highly interconnected nodes that form a rich club. Rich club nodes were defined as nodes with a degree one standard deviation above the average degree of all nodes (van den Heuvel and Sporns 2011). The rich club consisted of 19 nodes (total: $n=116$ ) across both hemispheres (Right: angular gyrus, temporal sulcus, supplementary motor area, cerebellum, amygdala, precuneus, anterior cingulate cortex, inferior frontal lobe, superior parietal lobule; Left: anterior cingulate gyrus, Heschl's gyrus, inferior temporal lobe, superior frontal cortex, medial orbitofrontal cortex, cerebellum, see Figure 4).

There was no significant difference in node eccentricity, i.e. node-level path length, between rich club and peripheral nodes (Rich club mean=21.37, $\mathrm{SE}=0.586$; Peripheral mean $=20.77, \mathrm{SE}=1.372 ; \mathrm{t}(114)=0.43, p=0.67)$. There was no significant difference in clustering coefficient between rich club and peripheral nodes (Rich club: mean $=0.08$, $\mathrm{SE}=0.003$; Peripheral: mean $=0.1, \mathrm{SE}=0.013 ; \mathrm{t}(114)=-1.97, p=0.052)$.

\section{Knock-down of rich club nodes has a strong effect on $\boldsymbol{C}_{\boldsymbol{G}}$ and $\boldsymbol{L}_{\boldsymbol{G}}$}

In order to assess the importance of peripheral and rich club nodes on the relationship between brain organisation and education scores, simulated attacks were carried out. Knock-down of rich club nodes had a more pronounced effect on $C_{G}$ and $L_{G}$ compared to attacks on peripheral nodes or random attacks (see Figure 6a). The resulting graph measures were used to calculate Maths and Reading scores using the linear model derived from the empirical data. The results indicated that increases in $L_{G}$ associated with targeted attack on rich club nodes had the largest effect on the predicted maths and reading scores using the regression model based on the observed data (see Figure $6 \mathrm{~b})$. The results indicated that attack on rich club nodes had a larger effect on predicted reading and maths scores than attacks on peripheral nodes or random attacks. 

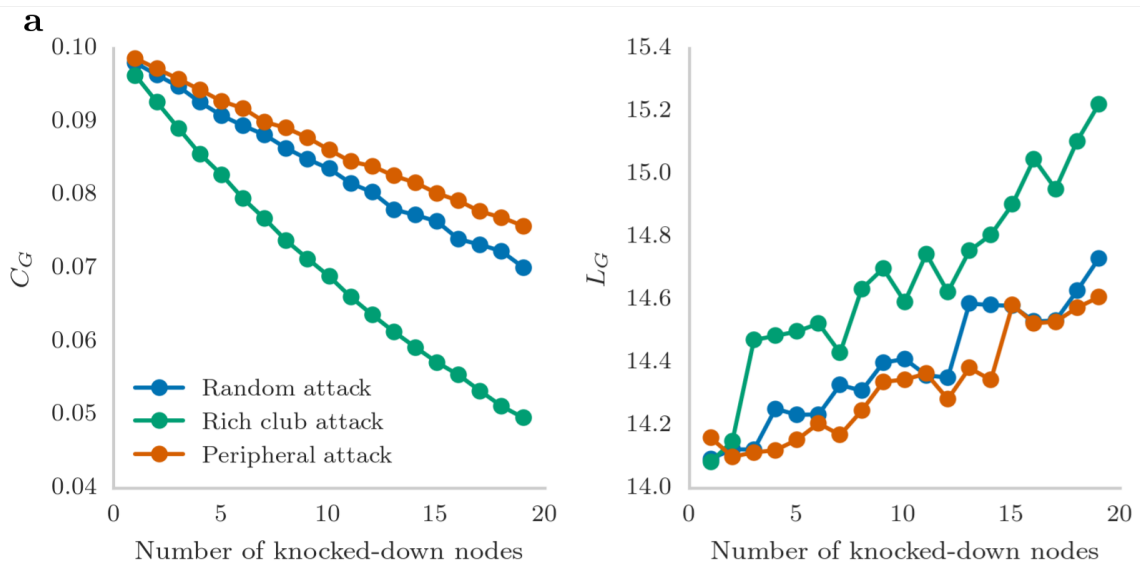

b
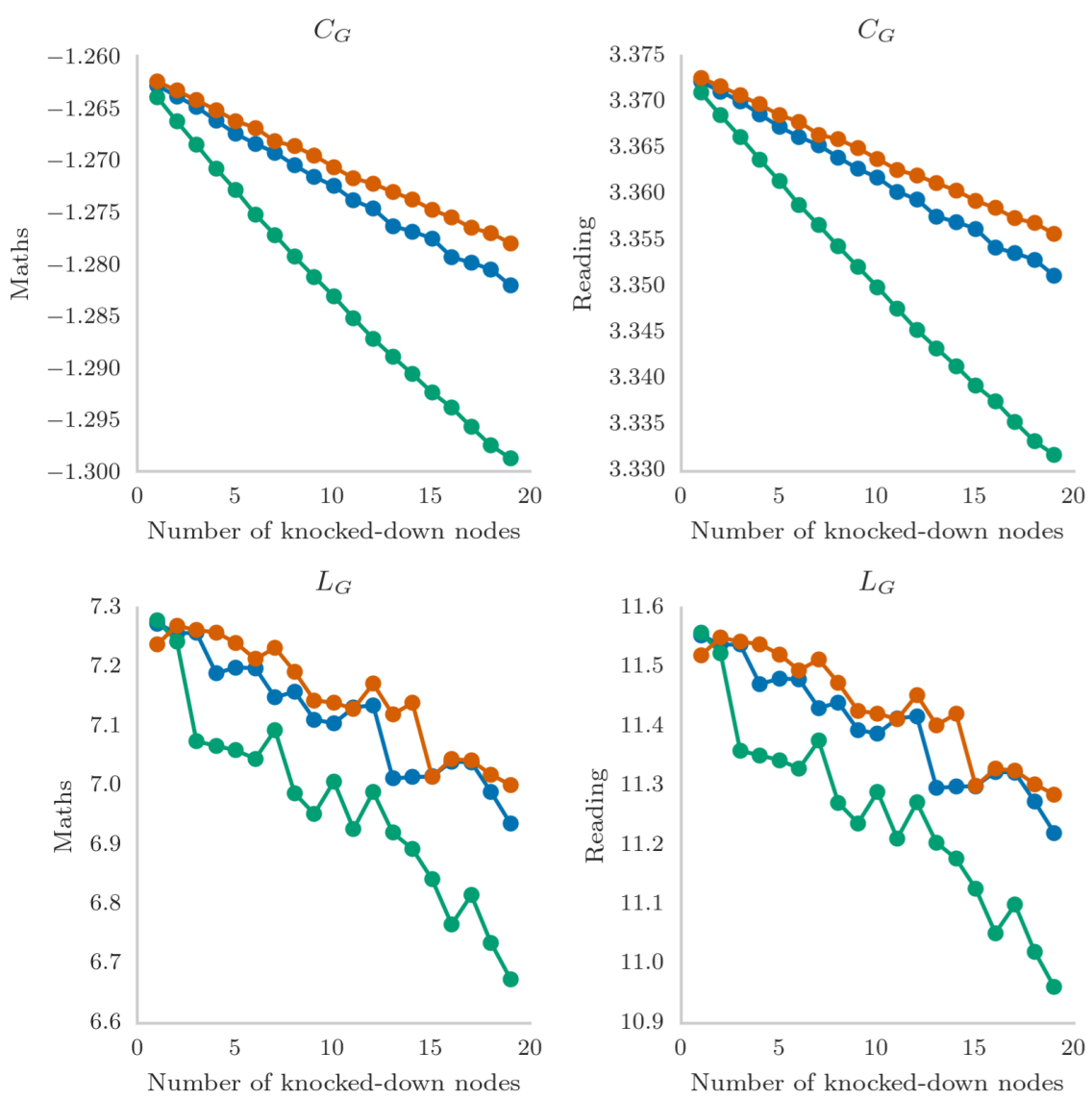

Figure 6: Results of simulated attack on rich club (green), peripheral (orange), and random nodes (blue). The edge weights associated with a targeted nodes in the group average network knocked-down and $L_{G}$ (left) and $C_{G}$ (right) were calculated in the resulting network. The number of knocked-down nodes was varied between 1 and 20 targeted nodes. $\boldsymbol{a}$ The results indicated that targeting rich club nodes resulted in the largest decrement in $C_{G}$ and increase in $L_{G}$ compared to attacks on peripheral or random nodes. $\boldsymbol{b}$ Increased path length following knock-down of rich club nodes resulted in the largest predicted decrement in Maths 
and Reading performance. Units are expressed as standard units relative to the mean and standard deviation of the empirical data.

\section{Development of structural brain organisation explains between 5 and $10 \%$ of age-related improvements in Maths and Reading scores}

Both maths and reading scores as well as graph metrics are likely to change with age. Therefore, the relationship between these measures and participant age was assessed in linear regression models. Reading and maths scores showed significant positive effects of age (model: $Y_{\text {Reading } / \text { Maths }}=\beta_{\text {age }} X_{\text {age }}+\beta_{\text {Intercept }}+\epsilon$; Reading: $\mathrm{F}(57)=52.91, p<0.001$, $R^{2}=0.48, \quad \beta_{\text {age }}=0.7, p<0.001$; Maths: $\mathrm{F}(88)=85.60, p<0.001, R^{2}=0.56, \beta_{\text {age }}=0.74$, $p<0.001)$. For graph measures, a significant positive relationship was found between age and $C_{G}$ (model: $Y_{C_{G} / L_{G}}=\beta_{\text {age }} X_{\text {age }}+\beta_{I C V} X_{I C V}+\beta_{\text {Displacement }} X_{\text {Displacement }}+\beta_{D_{G}} X_{D_{G}}+$ $\left.\beta_{\text {Intercept }}+\epsilon ; \mathrm{F}(88)=9.406, p<0.001, R^{2}=0.31, \beta_{\text {age }}=0.38, p<0.001\right)$, while a significant negative effect was indicated for $L_{G}\left(\mathrm{~F}(54)=14.40, \quad p<0.001, R^{2}=0.41, \quad \beta_{\text {Age }}=-0.52\right.$, $p<0.001)$.

In order to further investigate the relationship between age, graph measures, academic attainment scores, the amount of variance in attainment scores explained by age was compared between the original data and after regressing the effect of graph measures from attainment scores. A bootstrap procedure with 100 random selections was used to calculate confidence intervals for $R^{2}$. For maths, the amount of variance explained by age dropped from $85.7 \%(\mathrm{SE}=0.508)$ in the original data to $78.84 \%(\mathrm{SE}=0.636)$ when $C_{G}$ was controlled $(\mathrm{t}(24)=8.43, p<0.001)$. Similarly, variance explained by age was reduced from $86.6 \%$ ( $\mathrm{SE}=0.479)$ to $74.34 \%(\mathrm{SE}=0.844)$ when $L_{G}$ was held constant $(\mathrm{t}(24)=12.64$, $p<0.001$ ). For reading, variance explained by age went from $84.1 \%$ ( $\mathrm{SE}=0.519)$ to $77.72 \%(\mathrm{SE}=0.694)$ for $C_{G}(\mathrm{t}(24)=7.7, p<0.001)$, and from $83.54 \% \quad(\mathrm{SE}=0.498)$ to $72.93 \%(\mathrm{SE}=0.675)$ for $L_{G}(\mathrm{t}(24)=11.87, p<0.001)$. These findings indicate that between 5 and $12 \%$ of age-related improvements in Maths and Reading performance are attributable to developmental changes in structural brain connectivity.

\section{Differences in $C_{G}$ and $L_{G}$ with age are more closely related to rich club nodes}

Separate assessment of graph metrics related to rich club $\left(C_{\text {richclub }}, L_{\text {richclub }}\right)$ and peripheral nodes $\left(C_{\text {periphery }}, L_{\text {periphery }}\right)$ indicated a stronger relationship between $C_{\text {richclub }}$ and age compared to $C_{\text {periphery }}\left(C_{\text {richclub }}: \beta_{\text {age }}=0.51, p=0.001 ; C_{\text {periphery }}=0.43\right.$, $p=0.025)$. The relationship between age and $L$ was not significant after controlling for ICV, Movement, and $D_{G}\left(L_{\text {richclub }}: \beta_{\text {age }}=-0.01, \quad p=0.955 ; \quad L_{\text {periphery }}: \beta_{\text {age }}=0.18\right.$, 
$p=0.107)$. These results suggest that age-related changes in $C_{G}$ are more strongly driven by changing connections of rich club nodes.

\section{Comparison of lower and higher performing groups using voxel-wise statistics shows no group difference}

In order to contrast the connectome approach, the data of the current study were also analysed using the more conventional approach from developmental cognitive neuroscience in which participants are grouped by abilities rather than considered in terms of a continuum of performance. Lower and higher ability groups were defined relative to the group median of scores for reading and maths. There were groups of 45 children with low maths (mean=74.02, SE: 1.456 ), 43 with typical maths (mean=100.42, SE: 2.054$), 44$ with low reading (mean=73.14, SE: 1.41), and 44 with typical reading (mean=101.36, SE: 1.503; all age-standardised scores). The maths groups did not differ in age (Independent sample t-test: mean $_{\text {lower }}=113.27 \quad S E_{\text {lower }}=3.474$; mean $\left._{\text {higher }}=114.95, S E_{\text {higher }}=4.861 ; t(87)=-0.28, p=0.777\right)$. However, children in the reading deficit group were significantly younger than children in the typical reading group $\left(\right.$ mean $_{\text {lower }}=107.27, S E_{\text {lower }}=3.279 ;$ mean $_{\text {higher }}=120.91, S E_{\text {higher }}=4.723 ; t(87)=-$ 2.37, $p=0.02)$. Voxel-wise comparison of FA values between the groups with a commonly used method in diffusion imaging, i.e. tract-based spatial statistics, did not indicate any significant differences between the groups (see Figure 7). 

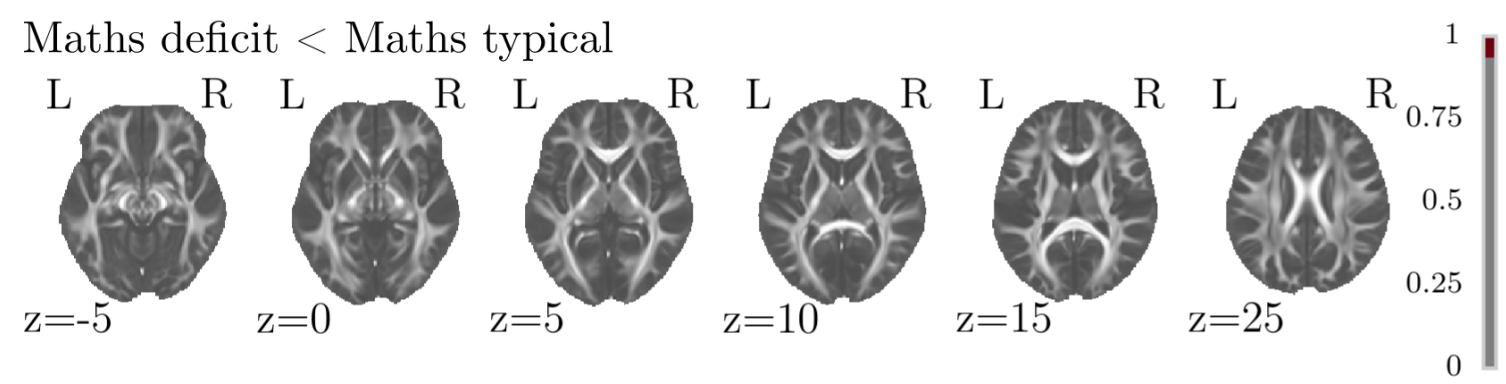

Maths typical $<$ Maths deficit
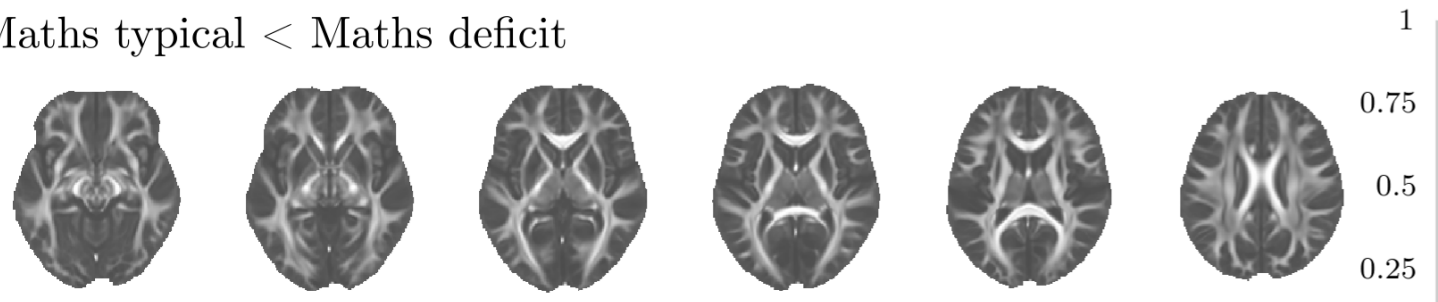

Reading deficit $<$ Reading typical
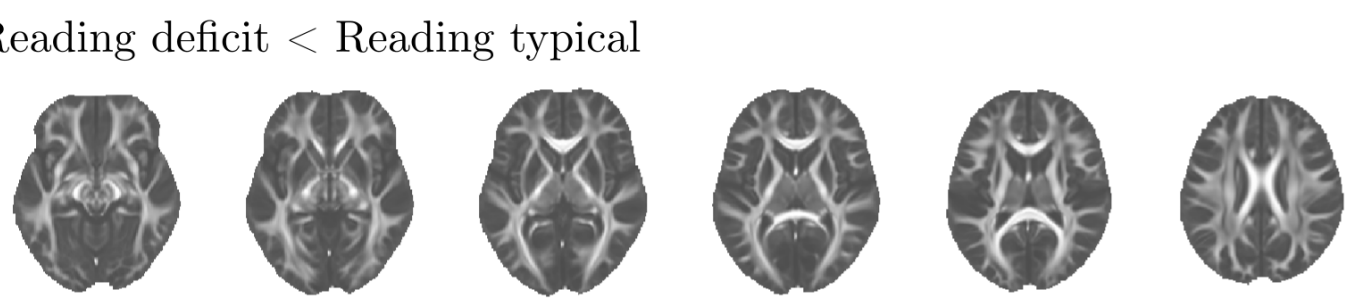

\section{5 \\ 0.25}

Reading typical $<$ Reading deficit
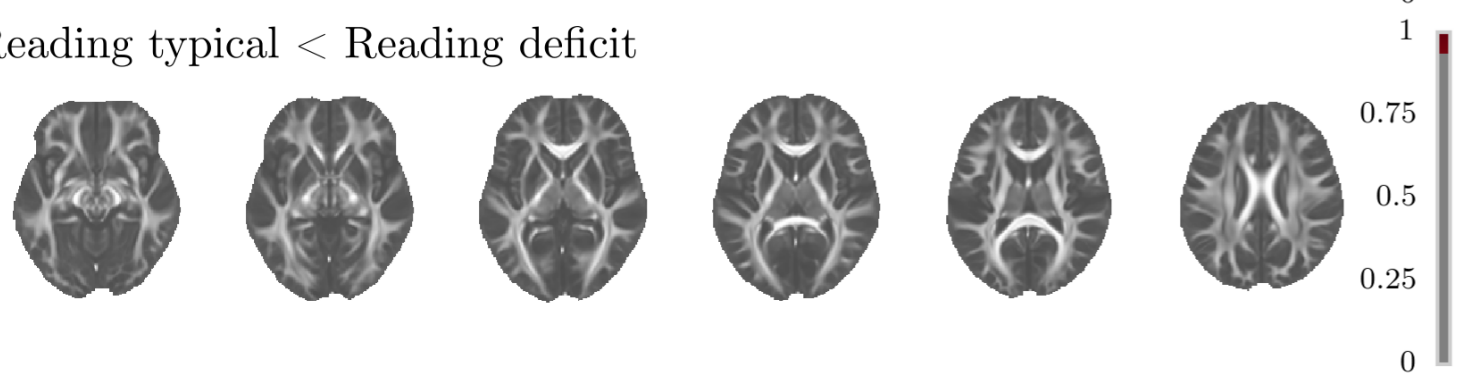

Figure 7: Comparison of deficit and typical groups based on median splits of the agestandardised maths and reading scores with tract-based spatial statistics (TBSS) indicated no clusters of significantly different FA values between the groups. The colour bars indicate 1-p thresholded for a significance level of $\alpha=0.05$. 


\section{Discussion}

The current study investigated the relationship between white matter organisation and literacy and numeracy development in childhood. We included a large sample of children with varying performance levels, including children with learning deficits in reading and/or maths. White matter connections in broad clusters were associated with learning scores for both reading and maths that extended well beyond focal differences in fronto-temporal and fronto-parietal regions that had been previously implicated (Carter et al. 2009, Rollins et al. 2009, Odegard et al. 2009, Matejko et al. 2013, Kucian et al. 2013, Beek et al. 2014). Furthermore, better reading and maths scores were related to two key measures of global brain network organisation: shorter path length and higher clustering. A simulated node knock-down showed that this relationship was most strongly mediated by highly connected hub nodes. Between 5 and $10 \%$ of improvement in reading and maths ability are attributable to shortening path length and increasing clustering in the white matter network with age, which was mostly driven by hub nodes.

The broad association between brain organisation and literacy and numeracy stands in apparent contrast to previous published findings that report focal differences in groups with learning deficits. The reason for this apparent disparity may stem from our contrasting approaches: case-control designs focus on selective deficits rigidly matched for other cognitive and environmental differences. This can give the impression of relative purity of problems in reading and maths, and standard voxel-wise statistical approaches tend to emphasise the restricted overlap across cases. The value of this complimentary approach is highlighted by submitting the same data to a more traditional approach - comparing typical and deficit groups using a voxel-wise comparison method. Although our groups differed substantially in their performance levels and the samples were large by comparison with many studies in the literature, this approach did not indicate any differences in white matter organisation for either literacy or numeracy. In contrast, network-based statistics indicate an association between learning scores and connections beyond established fronto-temporal and frontoparietal connections, including interhemispheric, cortico-subcortical, and corticocerebellar connections, in a large sample of children that are more representative of children with maths and reading difficulties in a school setting. A network approach can therefore uncover small differences that in sum lead to effects on overall brain organisation, which cannot be captured using a more traditional voxel-wise statistical approach. The findings of the current analysis indicate that these broader differences in brain organisation commonly contribute to the aetiology of specific learning difficulties 
A network science approach also allows us to test how differences across individual tracts contribute to broader differences in brain organisation and efficiency. Small-world organisation with high local connectivity and some long-range connection is thought to be central for optimal information transfer and minimal wiring cost (Bullmore and Sporns 2009, Watts and Strogatz 1998, Bullmore and Sporns 2012). This organisation is present from early in development and can be detected throughout the lifespan (Tymofiyeva et al. 2013, Huang et al. 2013, Dennis et al. 2013, Vértes and Bullmore 2014, Collin and van den Heuvel 2013). Small-world organisation can be characterised through graph measures. Short characteristic path length indicates that information between two nodes can be exchanged by traversing only few edges (Fornito, Zalesky, and Bullmore 2016), i.e. information transfer is faster, more direct, and less prone to noise interference (Bullmore and Sporns 2009). A decrease in characteristic path length, or increase in global efficiency which is inversely related to characteristic path length (Rubinov and Sporns 2010), as observed in the current study has been consistently reported in developmental studies (Hagmann et al. 2010, Yap et al. 2011, Fan et al. 2011, Dennis et al. 2013, Huang et al. 2013). Individual differences in characteristic path length in adults have been found to be highly heritable (Bohlken et al. 2014) and relate to cognitive abilities (van den Heuvel et al. 2009, Koenis et al. 2015). Age-related decreases in characteristic path length in the current study predicted literacy and numeracy scores. Thus increasing ease of information transfer in the structural brain network with age relates to improvements in literacy and numeracy as children grow-up and progress through school.

Another commonly used measure to characterise brain network organisation is the clustering coefficient, which is linked to network segregation. It quantifies the degree of local connectivity between neighbouring nodes. Reports about age-related changes in clustering coefficient are mixed with some studies reporting decreases in clustering (Hagmann et al. 2010, Dennis et al. 2013, Tymofiyeva et al. 2013), while other studies find increases (Wierenga et al. 2015, Huang et al. 2013). Discrepancies may be explained by methodological differences between the studies, specifically the metric used to express connection strength. Studies with streamline counts or weighted streamline counts found decreases of clustering coefficient with age, while studies with diffusion metric-weighted networks like the current study report increased clustering. Streamline measure may be more influenced by reduced connection likelihood with increasing distance as the brain grows. In contrast, networks based on diffusion metrics follow the developmental tendency of increasing fractional anisotropy with age (Imperati et al. 2011, Westlye et al. 2009), i.e. increasing weight in the connectivity matrix leads to a higher global clustering coefficient. The findings of the current study suggest that higher connectivity between neighbouring nodes with age relates to improvements in literacy and numeracy. 
Optimal organisation in brain networks has been found to depend on the presence of a smaller number of highly connected nodes (van den Heuvel et al. 2012) termed the rich club. This rich club is thought to be highly important for computational capacity (Senden et al. 2014) and is implicated across a range of adult and developmental disorders (Crossley et al. 2014). Rich club organisation is already established in the prenatal brain (Ball et al. 2014) and persists over childhood (Grayson et al. 2014) and adolescence into adulthood (Baker et al. 2015). Simulated knock-down in the current study suggested that knock-down of rich club nodes had a higher impact on path length and clustering compared to random or peripheral attacks. Since path length and clustering coefficient were found to be predictive of maths and reading scores, we conclude that rich club nodes are central for establishing a network architecture that can support literacy and numeracy optimally.

The association between literacy, numeracy, and brain organisation mirrors reported associations between cognitive functions and optimal network structure. For instance, van den Heuvel and colleagues (2009) found that shorter characteristic path length was associated with higher scores on an intelligence scale. This may indicate that optimal network architecture supports both better general cognitive function as well as higher capacity to learn. Alternatively, optimal network architecture may support particular cognitive abilities that are strongly linked to performance across a range of tasks, e.g. executive functions. Such an intermediate level of explanation could provide a fruitful avenue for future investigations.

The association between longer path length, reduced influence of hub nodes, and lower literacy and numeracy scores also resembles findings of atypical brain network organisation following early insults like preterm birth, hypoxia-ischaemia, and intrauterine growth restriction (Batalle et al. 2012, Pandit et al. 2013). Further, similar alterations in network organisation were also linked to common genetic variants associated with neurodevelopmental disorders like autism and ADHD (Dennis et al. 2011, Hong et al. 2014). This may suggest that various developmental constraints converge on sub-optimal brain network organisation via various mechanisms and that these differences in brain network organisation manifest in cognitive and behavioural symptoms commonly observed in developmental disorders.

In conclusion, previous studies about structural brain correlates of literacy and numeracy development suggested the involvement of a limited set of regions and their connections that are specifically linked to aspects of task-relevant processing. Using a complimentary approach that surveyed literacy and numeracy across a range of abilities and applying a network-analytic approach, the current study found that global organisation of the white matter network contributes to literacy and numeracy 
improvements with age. These results suggest that large-scale neural systems and their interaction play a role in the literacy and numeracy development in childhood. 


\section{References}

Avants, B., \& Gee, J. C. 2004. Geodesic estimation for large deformation anatomical shape averaging and interpolation. NeuroImage, 23, S139- S150. doi: 10.1016/j.neuroimage.2004.07.010

Baker, S. T. E., Lubman, D. I., Yucel, M., Allen, N. B., Whittle, S., Fulcher, B. D., \& Zalesky, A. 2015. Developmental changes in brain network hub connectivity in late adolescence. Journal of Neuroscience, 35 (24), 9078- 9087. doi: 10.1523/JNEUROSCI.5043-14.2015

Ball, G., Aljabar, P., Zebari, S., Tusor, N., Arichi, T., Merchant, N., Robinson, E. Ogundipe, D. Rueckert, A. D., \& Edwards, S. J. 2014. Rich-club organization of the newborn human brain. Proceedings of the National Academy of Sciences, 111(20), 7456-7461. doi: $10.1073 /$ pnas. 1324118111

Batalle, D., Eixarch, E., Figueras, F., Munoz-Moreno, E., Bargallo, N., Illa, M., Acosta-Rojas, R., Amat-Roldan, I., \& Gratacos E. 2012. Altered small-world topology of structural brain networks in infants with intrauterine growth restriction and its association with later neurodevelopmental outcome. NeuroImage, 60(2), 1352-1366. doi: 10.1016/j.neuroimage.2012.01.059

Bathelt, J., Gathercole, S. E., Johnson, A., \& Astle, D. E. 2016. Changes in brain morphology and working memory capacity over childhood. bioRxiv, 069617, doi: https://doi.org/10.1101/069617

Beek, L. V., Ghesquiere, P., Lagae, L., \& Smedt, B. D. 2014. Left fronto-parietal white matter correlates with individual differences in children's ability to solve additions and multiplications: A tractography study. NeuroImage, 90, 117-127. doi: 10.1016/j.neuroimage.2013.12.030

Bohlken, M. M., Mandl, R. C., Brouwer, R. M., Heuvel, M. P. van den, Hedman, A. M., Kahn, R. S., \& Hulshoff Pol, H. E. 2014. Heritability of structural brain network topology: A DTI study of 156 twins. Human Brain Mapping, 35(10), 5295-5305. doi: 10.1002/hbm.22550

Bullmore, E., \& Sporns, O. 2009. Complex brain networks: graph theoretical analysis of structural and functional systems. Nature Reviews Neuroscience, 10(3), 186-198. doi: $10.1038 / \operatorname{nrn} 2575$

Bullmore, E., \& Sporns, O. 2012. The economy of brain network organization. Nature Reviews Neuroscience. doi:10.1038/nrn3214

Carter, J. C., Lanham, D. C., Cutting, L. E., Clements-Stephens, A. M., Chen, X., Hadzipasic, M., Kim, J., Denckla, M. B., \& Kaufmann, W. E. 2009. A dual DTI approach to analyzing white matter in children with dyslexia. Psychiatry Research: Neuroimaging, 172(3), 215-219. doi: $10.1016 /$ j.pscychresns.2008.09.005 
Collin, G., \& Heuvel, M. P. van den. 2013. The ontogeny of the human connectome: Development and dynamic changes of brain connectivity across the life span. The Neuroscientist, 19(6), 616-628. doi: 10.1177/1073858413503712

Coupe, P., Yger, P., Prima, S., Hellier, P., Kervrann, C., \& Barillot, C. 2008. An optimized blockwise nonlocal means denoising filter for 3-D Magnetic Resonance Images. IEEE Transactions on Medical Imaging, 27(4), 425-441. doi: 10.1109/TMI.2007.906087

Crossley, N. A., Mechelli, A., Scott, J., Carletti, F., Fox, P. T., McGuire, P., \& Bullmore, E. T. 2014. The hubs of the human connectome are generally implicated in the anatomy of brain disorders. Brain, 137(8), 2382-2395. doi: 10.1093/brain/awu132

Dell'Acqua, F., \& Catani, M. 2012. Structural human brain networks: hot topics in diffusion tractography. Current Opinion in Neurology, 25(4):375-83. doi: 10.1097/WCO.0b013e328355d544.

Dennis, E. L., Jahanshad, N., McMahon, K. L., Zubicaray, G. I. de, Martin, N. G., Hickie, I. B., Toga, A. W., Wright, M. J., \& Thompson, P. M. 2013. Development of brain structural connectivity between ages 12 and 30: A 4-Tesla diffusion imaging study in 439 adolescents and adults. NeuroImage, 64, 671-684. doi: 10.1016/j.neuroimage.2012.09.004

Dennis, E. L., Jahanshad, N., Rudie, J. D., Brown, J. A., Johnson, K., McMahon, K. L., de Zubicaray, G. I., Montgomery, G., Martin, N. G., Wright, M. J., Bookheimer, S. Y., Dapretto, M., Toga, A. W., \& Thompson, P. M. 2011. Altered structural brain connectivity in healthy carriers of the autism risk gene CNTNAP2. Brain Connectivity, 1(6), 447-459. doi:10.1089/brain.2011.0064

Fan, Y., Shi, F., Smith, J. K., Lin, W., Gilmore, J. H., \& Shen, D. 2011. Brain anatomical networks in early human brain development. NeuroIm- age, 54(3), 1862-1871. doi: 10.1016/j.neuroimage.2010.07.025

Fornito, A., Zalesky, A., \& Breakspear, M. 2015. The connectomics of brain disorders. Nature Reviews Neuroscience, 16(3), 159-172. doi: 10.1038/nrn3901

Fornito, A., Zalesky, A., \& Bullmore, E. 2016. Fundamentals of brain network analysis. Amsterdam, Netherlands: Academic Press - Elsevier.

Garyfallidis, E., Brett, M., Amirbekian, B., Rokem, A., Walt, S. van der, Descoteaux, M., \& Nimmo-Smith, I. 2014. Dipy a library for the analysis of diffusion MRI data. Front. Neuroinform., 8. doi: 10.3389/fninf.2014.00008

Gillberg, C. 2010. The ESSENCE in child psychiatry: Early Symptomatic Syndromes Eliciting Neurodevelopmental Clinical Examinations. Research in Developmental Disabilities, 31(6), 1543-1551. doi: 10.3389/fninf.2014.00008 
Grayson, D. S., Ray, S., Carpenter, S., Iyer, S., Dias, T. G. C., Stevens, C., Iyer, S., Costa Dias, T. G., Stevens, C., Nigg, J. T., \& Fair, D. F. 2014. Structural and Functional Rich Club Organization of the Brain in Children and Adults. PLoS ONE, 9(2), e88297. doi: 10.1371/journal.pone.0088297

Hagmann, P., Sporns, O., Madan, N., Cammoun, L., Pienaar, R., Wedeen, V. J., Meuli, R., Thiran, J.- P. \& Grant. P. E. 2010. White matter maturation reshapes structural con- nectivity in the late developing human brain. Proceedings of the National Academy of Sciences, 107(44), 19067-19072. doi: 10.1073/pnas.1009073107

Heuvel, M. P. van den, Kahn, R. S., Goni, J., \& Sporns, O. 2012. High- cost high-capacity backbone for global brain communication. Proceedings of the National Academy of Sciences, 109(28), 11372-11377. doi: 10.1073/pnas.1203593109

Heuvel, M. P. van den, \& Sporns, O. 2011. Rich-Club Organization of the Human Connectome. Journal of Neuroscience, 31(44), 15775-15786. doi: 10.1523/JNEUROSCI.3539-11.2011

Heuvel, M. P. van den, Stam, C. J., Kahn, R. S., \& Pol, H. E. H. 2009. Efficiency of Functional Brain Networks and Intellectual Performance. Journal of Neuroscience, 29(23), 7619-7624. doi: $10.1073 /$ pnas. 1203593109

Hong, S.-B., Zalesky, A., Park, S., Yang, Y.-H., Park, M.-H., Kim, B., Song, I.-C., Sohn, C.-H., Shin, M.-S., Kim, B.-N., Cho, S.-C., Kim, J.-W. 2014. COMT genotype affects brain white matter pathways in attention-deficit/hyperactivity disorder. Human Brain Mapping, 36 (1), 367-377. doi: 10.1002/hbm.22634

Huang, H., Shu, N., Mishra, V., Jeon, T., Chalak, L., Wang, Z. J., Rollins, N., Gong, G., Cheng, H., Peng, Y., Dong, Q., \& He, Y. 2013. Development of Human Brain Structural Networks Through Infancy and Childhood. Cerebral Cortex, 25(5), 1389-1404. doi: 10.1093/cercor/bht335

Imperati, D., Colcombe, S., Kelly, C., Martino, A. D., Zhou, J., Castellanos, F. X., \& Milham, M. P. 2011. Differential Development of Human Brain White Matter Tracts. PLoS ONE, 6(8), e23437. doi: 10.1371/journal.pone.0023437

Johnson, M. H. 2003. Development of human brain functions. Biological Psychiatry, 54(12), 1312-1316. doi: 10.1016/S0006-3223(03)00426-8

Kucian, K. \&Aster, M. von 2014. Brain correlates of numerical disabilities. In M. S. Cohen \& A. Dowker (Eds.), Oxford handbook of numerical cognition. Oxford: Oxford University Press.

Karmiloff-Smith, A. 1998. Development itself is the key to understanding developmental disorders. Trends in Cognitive Sciences, 2(10), 389-398.

Khundrakpam, B. S., Reid, A., Brauer, J., Carbonell, F., Lewis, J., Ameis, S., Karama, S., Lee, J., Chen, Z., Das, S., Evans, A. C., Ball, W. S., Byars, A. W., Schapiro, M., Bommer, W., Carr, A., German, A., Dunn, S., Rivkin, M. J., Waber, D., Mulkern, R., Vajapeyam, S., Chiverton, A., Davis, P., Koo, J., Marmor, J., Mrakotsky, C., Robertson, R., McAnulty, G., Brandt, M. 
E., Fletcher, J. M., Kramer, L. A., Yang, G., McCormack, C., Hebert, K. M., Volero, H., Botteron, K., McKinstry, R. C., Warren, W. Nishino, T., Robert Almli, R., Todd, R., Constantino, R., McCracken, J. T, Levitt, J., Alger, J., O, J., Toga, A., Asarnow, R., Fadale, D., Heinichen, L., Ireland, C., Wang, D.-J., Moss, E., Zimmerman, R. A., Bintliff, B., Bradford, R., Newman, J., Evans, A. C., Arnaoutelis, R., Pike, B. R., Collins, D. L., Leonard, G., Paus, T., Zijdenbos, A., Das, S., Fonov, V., Fu, L., Harlap, J., Leppert, I., Milovan, D., Vins, D., Zeffiro, J., Van Meter, J., Lange, N.,Froimowitz, M. P., Botteron, K., Almli, C. R., Rainey, C., Henderson, S., Nishino, T., Warren, W., Edwards, J. L., Dubois, D., Smith, K., Singer, T., Wilber, A. A., Pierpaoli, C., Basser, P. J., Chang, L.-C., Koay, C. G., Walker, L., Freund, L., Rumsey, J., Baskir, L., Stanford, L., Sirocco, K., Gwinn-Hardy, K., Spinella, G., McCracken, J. T., Alger, J. R., Levitt, J., \& O, J. 2012. Developmental Changes in Organization of Structural Brain Networks. Cerebral Cortex, 23(9), 2072-2085. doi: 10.1093/cercor/bhs187

Koenis, M. M., Brouwer, R. M., Heuvel, M. P. van den, Mandl, R. C., Soelen, I. L. van, Kahn, R. S., Boomsma, D. I., Hulshoff Pol, H. E. 2015. Development of the brain's structural network efficiency in early adolescence: A longitudinal DTI twin study. Human Brain Mapping, 36(12), 4938-4953. doi: 10.1002/hbm.22988

Konrad, K., \& Eickhoff, S. B. 2010. Is the ADHD brain wired differently? A review on structural and functional connectivity in attention deficit hyperactivity disorder. Human Brain Mapping, 31(6), 904-916. doi: 10.1002/hbm.21058

Kovas, Y., Haworth, C., Harlaar, N., Petrill, S., Dale, P., \& Plomin, R. 2007. Overlap and specificity of genetic and environmental influences on mathematics and reading disability in 10year-old twins. Journal of Child Psychology and Psychiatry, 48(9), 914-922. doi: 10.1111/j.14697610.2007.01748..x

Kucian, K., Ashkenazi, S. S., Hanggi, J., Rotzer, S., J“ancke, L., Martin, E., et al. (2013). Developmental dyscalculia: a dysconnection syndrome? Brain Structure and Function.

Landerl, K., \& Moll, K. (2010). Comorbidity of learning disorders: prevalence and familial transmission. Journal of Child Psychology and Psychiatry, 51(3), 287-294. doi: 10.1111/j.14697610.2009.02164.x

Larson, K., Russ, S. A., Kahn, R. S., \& Halfon, N. (2011) Patterns of comorbidity functioning, and service use for us children with ADHD, 2007. Pediatrics, 127(3), 462-470. doi: 10.1542 / peds.2010-0165

Martino, A. D., Fair, D. A., Kelly, C., Satterthwaite, T. D., Castellanos, F. X., Thomason, M. E., Craddock, R. C., Luna, B., Leventhal, B. L., Zuo, X.-N., Milham, M. P. 2014. Unraveling the Miswired Connectome: A Developmental Perspective. Neuron, 83(6), 1335-1353. doi: 10.1016/j.neuron.2014.08.050 
Matejko, A. A., Price, G. R., Mazzocco, M. M., \& Ansari, D. 2013. Individual differences in left parietal white matter predict math scores on the Preliminary Scholastic Aptitude Test. NeuroImage, 66, 604-610. doi: 10.1016/j.neuroimage.2012.10.045

Odegard, T. N., Farris, E. A., Ring, J., McColl, R., \& Black, J. 2009. Brain connectivity in nonreading impaired children and children diagnosed with developmental dyslexia. Neuropsychologia, 47(8-9), 1972-1977. doi: 10.1016/j.neuropsychologia.2009.03.009

Pandit, A. S., Robinson, E., Aljabar, P., Ball, G., Gousias, I. S., Wang, Z., et al. (2013). WholeBrain mapping of structural connectivity in infants reveals altered connection strength associated with growth and preterm birth. Cerebral Cortex, 24(9), 2324-2333.

Pannek, K., Hatzigeorgiou, X., Colditz, P. B., \& Rose, S. 2013. Assessment of structural connectivity in the preterm brain at term equivalent age using diffusion MRI and T2 relaxometry: A network-based analysis. PLoS ONE, 8(8), e68593. doi: 10.1371/journal.pone.0068593

Pascual-Leone, A., Amedi, A., Fregni, F., \& Merabet, L. B. 2005. The plastic human brain cortex. Annu. Rev. Neurosci., 28 (1), 377-401. doi: 10.1146/annurev.neuro.27.070203.144216

Pennington, B. F., \& Bishop, D. V. 2009. Relations Among Speech Language, and Reading Disorders. Annual Review of Psychology, $60 \quad$ (1), 283- 306 . doi: 10.1146/annurev.psych.60.110707.163548

Peterson, R. L., \& Pennington, B. F. 2015. Developmental Dyslexia. Annual Review of Clinical Psychology, 11(1), 283-307. doi: 10.1146/annurev-clinpsy-032814-112842

Qi, S., Meesters, S., Nicolay, K., Haar Romeny, B. M., \& Ossenblok, P. 2015. The influence of construction methodology on structural brain network measures: A review. Journal of Neuroscience Methods, 253, 170-182. doi: 10.1016/j.jneumeth.2015.06.016

Ranpura, A., Isaacs, E., Edmonds, C., Rogers, M., Lanigan, J., Singhal, A., Clayden, J., Clark, C., Butterworth, B. 2013. Developmental trajectories of grey and white matter in dyscalculia. Trends in Neuroscience and Education, 2(2), 56-64. doi: 10.1016/j.tine.2013.06.007

Reus, M. A. de, \& Heuvel, M. P. van den. 2013. Estimating false positives and negatives in brain networks. NeuroImage, 70, 402-409. doi: 10.1016/j.neuroimage.2012.12.066

Rollins, N. K., Vachha, B., Srinivasan, P., Chia, J., Pickering, J., Hughes, C. W., \& Gimi, B. 2009. Simple developmental dyslexia in children: Alterations in diffusion-tensor metrics of white matter tracts at 3 T 1. Radiology, 251(3), 882-891. doi: 10.1148/radiol.2513080884

Rubinov, M., \& Sporns, O. 2010. Complex network measures of brain connectivity: Uses and interpretations. NeuroImage, 52(3), 1059-1069. doi: 10.1016/j.neuroimage.2009.10.003 
Senden, M., Deco, G., Reus, M. A. de, Goebel, R., \& Heuvel, M. P. van den. 2014. Rich club organization supports a diverse set of functional network configurations. NeuroImage, 96, 174182. doi: $10.1016 /$ j.neuroimage.2014.03.066

Shi, F., Wang, L., Peng, Z., Wee, C.-Y., \& Shen, D. 2013. Altered Modular Organization of Structural Cortical Networks in Children with Autism. PLoS ONE, 8(5), e63131. doi: 10.1371/journal.pone.0063131

Smith, S. M., Jenkinson, M., Johansen-Berg, H., Rueckert, D., Nichols, T. E., Mackay, C. E., Watkins, K. E., Ciccarelli, O., Cader, M. Z., Matthews, P. M., Behrens, T. E. J. 2006. Tractbased spatial statistics: Voxelwise analysis of multi-subject diffusion data. NeuroImage, 31(4), 1487-1505. doi: 10.1016/j.neuroimage.2006.02.024

Tournier, J.-D., Calamante, F., \& Connelly, A. 2012. MRtrix: Diffusion tractography in crossing fiber regions. Int. J. Imaging Syst. Technol., 22(1), 53-66. doi: 10.1002/ima.22005

Tymofiyeva, O., Hess, C. P., Ziv, E., Lee, P. N., Glass, H. C., Ferriero, D. M., Barkovich, A. J., \& Xu, D. 2013. A DTI-Based Template-Free Cortical Connectome Study of Brain Maturation. PLoS ONE, 8(5), e63310. doi: 10.1371/journal.pone.0063310

Vertes, P. E., \& Bullmore, E. T. 2014. Annual Research Review: Growth connectomics - the organization and reorganization of brain networks during normal and abnormal development. J Child Psychol Psychiatry, 56 (3), 299-320. doi: 10.1111/jcpp.12365

Watts, D. J., \& Strogatz, S. H. (1998). Nature, 393(6684), 440-442. doi: 10.1038/30918

Wechsler, D. (2005). Wechsler Individual Achievement Test - Second UK edition. Oxford, UK: Pearson Assessment.

Westlye, L. T., Walhovd, K. B., Dale, A. M., Bjornerud, A., Due-Tonnessen, P., Engvig, A., Grydeland, H., Tamnes, C. K., Ostby, Y., \& Fjell, A. M. 2009. Life-Span Changes of the Human Brain White Matter: Diffusion Tensor Imaging (DTI) and Volumetry. Cerebral Cortex, 20(9), 2055-2068. doi: 10.1093/cercor/bhp280

Wierenga, L. M., Heuvel, M. P. van den, Dijk, S. van, Rijks, Y., Reus, M. A. de, \& Durston, S. 2015. The development of brain network architecture. Human Brain Mapping, 37(2), 717-729. doi: $10.1002 / \mathrm{hbm} .23062$

Winkler, A. M., Ridgway, G. R., Webster, M. A., Smith, S. M., \& Nichols, T. E. 2014. Permutation inference for the general linear model. NeuroImage, 92, 381-397. doi: 10.1016/j.neuroimage.2014.01.060

Xia, M., Wang, J., \& He, Y. 2013. BrainNet Viewer: a network visualization tool for human brain connectomics. PLoS ONE, 8(7), e68910. doi: 10.1371/journal.pone.0068910 
Yap, P.-T., Fan, Y., Chen, Y., Gilmore, J. H., Lin, W., \& Shen, D. 2011. Development trends of white matter connectivity in the first years of life. PLoS ONE, 6(9), e24678. doi: 10.1371/journal.pone.0024678

Zalesky, A., Fornito, A., \& Bullmore, E. T. 2010. Network-based statistic: Identifying differences in brain networks. NeuroImage, 53(4), 1197-1207. doi: 10.1016/j.neuroimage.2010.06.041 